\title{
Minimização do Consumo de Energia de Dispositivos Sem Fio em Ambientes Industriais
}

\author{
Marcus de V. D. da Silva ${ }^{1}$, Gabriel Galdino ${ }^{1}$, Rafael L. Gomes ${ }^{1}$ \\ ${ }^{1}$ Universidade Estadual do Ceará (UECE) \\ \{marcus, gabriel.galdino, rafaelllgom\}larces.uece.br
}

\begin{abstract}
Resumo. Recentemente a era da Internet das Coisas Industrial (IIoT) emergiu a fim de garantir a produção eficiente e sustentável para o contexto de Industria 4.0. Uma abordagem promissora para implantar ambientes IIoT é o uso da tecnologia IEEE 802.11 como forma de comunicação sem fio. Contudo, a configuração usual da tecnologia IEEE 802.11 resulta em maior consumo de energia dos dispositivos IIoT, devido ao tamanho dos pacotes transmitidos. Dentro deste contexto, este artigo apresenta um método de compressão de dados para reduzir a quantidade de dados a serem transmitidos, com o objetivo de minimizar o consumo de energia dos dispositivos nesses cenários. Experimentos realizados em um cenário de teste real sugerem a redução do consumo de energia quando o método proposto é aplicado ao contexto IIoT.
\end{abstract}

\begin{abstract}
Recently the Industrial Internet of Things (IIoT) era arose to supports efficient and sustainable production in Industry 4.0 context. A promising approach to deploy IIoT environments is the usage of IEEE 802.11 as wireless communication technology. Nevertheless, the default configuration of IEEE 802.11 technology results in higher energy consumption of the IIoT devices, due to the size of the packets transmitted. Within this context, this paper presents a data compression method to reduce the amount of data to be transmitted, aiming to minimize the energy consumption of IIoT devices. Experiments performed in a real testbed suggest the reduction of energy consumption when the proposed method is applied in IIoT context.
\end{abstract}

\section{Introdução}

A evolução dos microcontroladores trouxe maior capacidade computacional e componentes eletrônicos mais baratos, proporcionando o aumento do número de dispositivos de comunicação. Com este aumento, foi possível o uso dos mesmos na automatização de tarefas em vários ambientes. Esse cenário criou uma nova onda de evolução, chamada Internet das Coisas (IoT) [Li et al. 2018b].

A partir do conceito de IoT surgiram diversos ambientes inteligentes com recursos e aplicações específicas, como Casas Inteligentes, Cidades Inteligentes e outros. Um desses ambientes é a Internet das Coisas Industrial (IIoT) para o contexto de Indústria 4.0 [Wollschlaeger et al. 2017]. A IIoT inclui a comunicação sem fio máquina a máquina (M2M) e industrial para apoiar uma produção eficiente e sustentável [Sisinni et al. 2018]. Assim, a IIoT oferece enorme eficácia e benefícios econômicos à instalação de sistemas, manutenção, confiabilidade e interoperabilidade [Long et al. 2018]. 
As aplicações IIoT geralmente exigem uma taxa de transferência relativamente pequena por nó, mas o tráfego agregado pode atingir valores altos. Além disso, a IIoT requer a interconexão de um grande número de dispositivos à Internet a baixo custo, com recursos de Hardware (HW) e de energia limitados (por exemplo, baterias de baixa capacidade). Essas características tornam a latência, o grande número de conexões, a eficiência energética, o custo, a confiabilidade e a segurança / privacidade questões cruciais a serem consideradas na IIoT [Sisinni et al. 2018]. Por exemplo, nas referências [Sisinni et al. 2018, Varghese and Tandur 2014], os autores consideram os seguintes requisitos para a IIoT: a duração da bateria é de 10 anos, 300.000 dispositivos conectados por gateway IIoT e suporte para taxas de tráfego de dados agregado em torno de 1 Gbps.

Em geral, os ambientes de IoT são compostos de dispositivos que usam comunicação sem fio de baixa largura de banda, como ZigBee (IEEE 802.15.4 Standard) ou Bluetooth Low Energy (BLE) [Davoli et al. 2017]. Essas tecnologias carecem de robustez para atender aos requisitos da IIoT quando comparadas à tecnologia IEEE 802.11 / WiFi. A potência de transmissão do IEEE 802.11 é maior e possui menor tempo de acesso, resultando em um cenário injusto em ambientes heterogêneos, visto que o IEEE 802.11 obtém prioridade de acesso ante o 802.15.4 [Talebi et al. 2018].

A partir disso, o uso da tecnologia IEEE 802.11 / WiFi em ambientes IIoT apresenta as seguintes vantagens [Sisinni et al. 2018, Varghese and Tandur 2014, Mesquita et al. 2018]: (I) dispositivos WiFi têm um custo menor que os dispositivos ZigBee; (II) o protocolo IEEE 802.11 já possui alternativas para problemas de segurança e escalabilidade; e, (III) o padrão IEEE 802.11 suporta a abordagem plug-and-play e um grande número de dispositivos conectados.

No entanto, a configuração usual da tecnologia IEEE 802.11 / WiFi resulta em maior consumo de energia dos dispositivos sem fio, devido ao tamanho dos pacotes transmitidos e de acordo com o Unidade Máxima de Transmissão (Maximum Transmission Unit - MTU) definido [Sharma et al. 2018, Björnson and Larsson 2018]. O tamanho do MTU interfere diretamente no acesso ao meio dos dispositivos, visto que o do pacote transmitido diretamente relacionado ao tempo de ocupação do meio [Datasheet 2015, Siu 2018], tanto que soluções como 6LoWPAN definem um MTU de 127 bytes para melhorar a capacidade de acesso ao meio dos dispositivos [Shelby and Bormann 2011, Olsson 2014]. O impacto da variação do MTU fica mais nítido em contexto de maior escala, como é o caso de Industria 4.0. Assim, o atual estado da arte da IIoT requer uma solução para permitir um menor consumo de energia para as transmissões de dispositivos sem fio tratando destes aspectos de transmissão de dados e MTU.

Dentro deste contexto, este artigo apresenta um método de compressão de dados para reduzir a quantidade de dados a serem transmitidos, habilitar a redução do MTU dos dispositivos e, consequentemente, minimizar o consumo de energia de dispositivos IIoT com base na tecnologia IEEE 802.11 / WiFi. Além disso, o método proposto aplica um algoritmo de compressão de baixa complexidade, a fim de reduzir o consumo de energia do dispositivo devido a execução da solução.

Experimentos foram realizados em um ambiente de teste real composto por vários dispositivos ESP8266 [Datasheet 2015, Kanakaris et al. 2019] usando o protocolo MQTT (Message Queuing Telemetry Transport), caracterizando um ambiente IIoT. Os experi- 
mentos visaram avaliar a capacidade do método proposto para melhorar a vida útil da bateria dos dispositivos sem fio IIoT, ou seja, o consumo de energia dos dispositivos variando o tamanho dos pacotes transmitidos e do MTU. Os resultados indicam que o uso da compressão de dados na IIoT baseada em IEEE 802.11/WiFi reduz o consumo de energia em cerca de $8 \%$ quando comparado com o uso padrão em ambientes IIoT.

Sendo assim, o artigo possui as seguintes contribuições: A) Avaliação dos aspectos de MTU e consumo de energia em cenário real; e, B) Adaptação da compressão com Huffman o contexto de Microcontroladores IoT, através da definição de uma árvore de acordo com a amostragem de caracteres plausíveis.

O restante deste artigo está organizado da seguinte forma: Seção 2 apresenta os trabalhos relacionados existentes. A seção 3 descreve o método de compressão de dados proposto, enquanto a seção 4 detalha os resultados dos experimentos realizados. Finalmente, a Seção 5 conclui o artigo e apresenta os trabalhos futuros.

\section{Trabalhos Relacionados}

A seguir, apresentamos os trabalhos relacionados à estratégias para economia de energia em Redes de Sensores Sem Fio (RSSF), IoT, IIoT. A tabela 1 resume os trabalhos descritos, enfatizando a diferença entre eles e nossa proposta. Na tabela 1, a coluna Contexto apresenta o ambiente em que o trabalho relacionado atua, enquanto as colunas Estratégia e Foco informam a estratégia aplicada e o objetivo da proposta dos artigos, respectivamente.

Biswas et al. [Biswas et al. 2018] propõem um algoritmo de coleta de dados de caminho mais curto distribuído para cobertura de alvo, com o objetivo de maximizar a vida útil da energia em RSSFs de múltiplos saltos e garantir eficiência energética e confiabilidade à rede. No entanto, este artigo não considera o volume de dados transmitidos, bem como as questões do contexto da IIoT.

Kai Li et al. [Li et al. 2018a] modelam um problema de otimização de agendamento para redes de sensores móveis para captação de energia, que maximiza a quantidade de dados coletados sob as restrições de qualidade do link de rádio e eficiência na captação de energia. Os autores apresentam um algoritmo heurístico para aproximar a solução ótima de agendamento em tempo polinomial. Contudo, os autores não consideram os problemas do ambiente IIoT e uma possível abordagem de compressão de dados.

Malleswari et al. [Malleswari et al. ] usam uma codificação de Huffman modificada para compressão de dados de segurança, melhorando a vida da bateria transmitindo dados compactados para consumir menos energia. Apesar de os autores aplicarem uma abordagem de compressão de dados, eles não avaliam a proposta em um ambiente de teste real e com base no contexto IIoT (por exemplo, aplicações Wifi), assim como não avaliam o impacto do processamento de compressão de dados no consumo de energia dos dispositivos.

Ertaul e Woodall [Ertaul and Woodall 2017] avaliam a implementação do software Grain, MICKEY e Trivium em um NodeMC para aplicações de IoT. O NodeMC está programado para aguardar a comunicação TCP em uma rede Wi-Fi para criptografar ou descriptografar texto usando essas cifras de fluxo leves, proporcionando um bom desempenho de taxa de transferência e baixo consumo de energia. No entanto, os autores 
não se concentram em estratégias para reduzir o consumo de energia na transmissão sem fio.

Zhetao Li et al. [Li et al. 2018b] focam em otimizar a estrutura de grupo de sensores sem fio para minimizar o atraso e o consumo de energia para a agregação convergente em ambientes verdes de IoT. Os autores propõem uma arquitetura de rede em grupos, permitindo a agregação de dados entre os grupos. Portanto, os nós dos sensores podem ser agendados em intervalos de tempo consecutivos para reduzir o número de transições de estado, atingindo consequentemente o objetivo de minimizar o atraso e o consumo de energia. Apesar do foco no consumo de energia, os autores não avaliam a proposta em um ambiente de teste real com dispositivos IIoT (várias simulações foram realizadas) e apenas realiza a agregação de dados (sem compressão).

Long et al. [Long et al. 2018] propõem um esquema de roteamento que aprimora o consumo de energia e o atraso de ponta-a-ponta (E2E) para sistemas IIoT em larga escala baseados no MAC IEEE 802.15.4a. O algoritmo proposto é direcionado a sistemas de grande escala, onde os dados são agregados através de diferentes grupos hierárquicos a caminho do coletor. Conduto, os autores não aplicam uma abordagem de compressão de dados e os problemas de transmissão sem fio não são considerados.

Tabela 1. Trabalhos Relacionados

\begin{tabular}{|l|l|l|l|}
\hline Referência & Contexto & Estratégia & $\begin{array}{l}\text { Objetivo } \\
\text { tribuídos de Dados Dis- }\end{array}$ \\
\hline [Biswas et al. 2018] & RSSF & $\begin{array}{l}\text { Eficiência } \\
\text { energética no } \\
\text { rastreamento de } \\
\text { alvos }\end{array}$ \\
\hline [Li et al. 2018a] & RSSF & $\begin{array}{l}\text { Algoritmo Heurístico } \\
\text { de Escalonamento }\end{array}$ & $\begin{array}{l}\text { Coleta de Dados e } \\
\text { de Energia }\end{array}$ \\
\hline [Malleswari et al. ] & RSSF & Compressão de Dados & $\begin{array}{l}\text { Aumentar a vida } \\
\text { útil da bateria }\end{array}$ \\
\hline [Tömösközi et al. 2017] & $\begin{array}{l}\text { Disposit. } \\
\text { Móveis }\end{array}$ & $\begin{array}{l}\text { Compressão de } \\
\text { Cabeçalho }\end{array}$ & $\begin{array}{l}\text { Avaliar do consumo } \\
\text { de energia }\end{array}$ \\
\hline [Ertaul and Woodall 2017] & IoT & $\begin{array}{l}\text { Wifi e Cifras de Fluxo } \\
\text { Leves }\end{array}$ & $\begin{array}{l}\text { Melhorar o rendi- } \\
\text { mento e a eficiência } \\
\text { energética }\end{array}$ \\
\hline [Li et al. 2018b] & IoT & $\begin{array}{l}\text { Arquitetura de Redes } \\
\text { em Cluster }\end{array}$ & $\begin{array}{l}\text { Reduzir o atraso e o } \\
\text { consumo de energia }\end{array}$ \\
\hline [Long et al. 2018] & IIoT & $\begin{array}{l}\text { Agregação de Da- } \\
\text { dos em Clusters } \\
\text { Hierárquicos }\end{array}$ & $\begin{array}{l}\text { Minimizar o con- } \\
\text { sumo de energia e o } \\
\text { atraso fim a fim. }\end{array}$ \\
\hline Método proposto & IIoT & $\begin{array}{l}\text { Compressão de dados } \\
\text { em IEEE 802.11/Wifi }\end{array}$ & $\begin{array}{l}\text { Baixo consumo de } \\
\text { energia }\end{array}$ \\
\hline
\end{tabular}

A partir do levantamento bibliográfico realizado, nota-se que nenhum artigo da literatura se concentrou no desenvolvimento de uma abordagem de compressão de dados que permite o uso de Wifi de baixa potência na IIoT e melhora a eficiência energética dos dispositivos IoT, que é o foco deste artigo. Os trabalhos encontrados na literatura focados 
em compressão possuem uma complexidade incompatível com o HW utilizado em IIoT e/ou aplicam compressão parcial (somente do cabeçalho) em dispositivos pessoais, que possuem capacidade computacional fora do contexto de IIoT.

\section{Proposta}

Tradicionalmente, os dispositivos (sensores e atuadores) em ambientes industriais usam comunicação cabeada, mas esse cenário mudou com o conceito de Indústria 4.0 [Wollschlaeger et al. 2017]. Tais ambientes exigem novos requisitos (como um grande número de conexões, eficiência energética, escalabilidade e outros [Sisinni et al. 2018]), o que compromete o uso da comunicação cabeada. A partir desse cenário, surgiu o paradigma IIoT com o objetivo de apoiar uma produção eficiente e sustentável [Long et al. 2018]. No entanto, o atual estado da arte da IIoT ainda não possui uma solução para permitir menor consumo de energia para transmissões de dispositivos sem fio usando IEEE 802.11 / WiFi, atendendo aos requisitos da indústria 4.0.

Este artigo propõe um método de compressão de dados para reduzir a quantidade de dados a serem transmitidos e habilitar a redução do MTU, com o objetivo de minimizar o consumo de energia de dispositivos IIoT com base na tecnologia IEEE 802.11/WiFi. A seguir, as subseções 3.1 e 3.2 descreverão os problemas de hardware e software considerados na proposta e o método de compressão de dados proposto, respectivamente.

\subsection{Aspectos de Hardware Relacionados a Indústria 4.0}

A IoT e a IIoT têm várias diferenças das perspectivas de aplicações e requisitos. A maioria dos cenários de IoT funciona no modelo Business to Consumer (B2C), uma vez que se concentra na provisão de serviços aos usuários finais, aprimorando questões mais relacionadas a Big Data, automação, entretenimento, etc. Por outro lado, a IIoT trabalha com o modelo Business to Business (B2B), ou seja, interação entre prestadores de serviços e produtos, com foco em custo financeiro, escalabilidade, consumo de energia e outros [Mumtaz et al. 2017]. Portanto, a IIoT requer mais regulamentação com requisitos específicos e padronização para hardware e software [Wollschlaeger et al. 2017].

Desde o início da implantação da IoT, a maioria dos dispositivos sem fio usa comunicação sem fio de baixa largura de banda (como o ZigBee), uma vez que esses dispositivos eram mais baratos que os dispositivos IEEE 802.11 / WiFi. No entanto, esse cenário mudou com o advento de dispositivos IEEE 802.11 / WiFi pequenos e baratos (como ESP8266-1 e ESP8266X / WEMOS [Kanakaris et al. 2019]), tornando-se uma solução adequada e promissora para os ambientes IIoT a fim de atender os requisitos da indústria 4.0 [Sisinni et al. 2018, Varghese and Tandur 2014].

Um requisito crucial para a IIoT é o consumo de energia dos dispositivos. O consumo de energia de um dispositivo IIoT $(E)$ em um determinado período de tempo $(t)$ pode ser considerado como na Equação 1 [Akintade et al. 2019], em que $T X, R X$, $C P U$ e $S$ são a quantidade de energia gasta na transmissão de dados, recepção de dados, processamento de dados e modo de hibernação, respectivamente. Da mesma forma, $t_{T X}, t_{R X}, t_{C P U}$ e $t_{S}$ são a quantidade de tempo que o dispositivo IIoT executou a transmissão, recepção, processamento de dados e estava no modo de hibernação, respectivamente. Sendo assim, $t$ é a soma de $t_{T X}, t_{R X}, t_{C P U}$ e $t_{S}$. 


$$
E(t)=T X * t_{T X}+R X * t_{R X}+C P U * t_{C P U}+S * t_{S}
$$

Por exemplo, os dispositivos ESP8266-1 e ESP8266X-WEMOS operam com voltagem de $80 \mathrm{~mA}$, sendo compatíveis com o modo de hibernação profunda (voltagem de 20 mu A com rádio e CPU parcialmente desativados), enquanto utilizam $15 \mathrm{~mA}$ para processamento de CPU, $56 \mathrm{~mA}$ para recepção de dados e $120 \mathrm{~mA}$ para transmissão de dados [Akintade et al. 2019].

Uma desvantagem do uso da tecnologia IEEE 802.11 / WiFi na IIoT é o consumo de energia para transmissão sem fio, devido ao tamanho dos pacotes e do MTU definido. A minimização da quantidade de dados transmitidos pelos dispositivos sem fio (carga útil) permite reduzir o MTU, consequentemente, o consumo de energia [Björnson and Larsson 2018]. O MTU precisa ser compatível com o tamanho da mensagem, pois um MTU mais baixo resulta em fragmentação de pacotes e um MTU mais alto gera uma dificuldade maior para acesso ao meio do que o necessário. Portanto, o atual estado da arte da IIoT requer uma solução para permitir essa redução de MTU e minimizar os dados transmitidos, bem como o baixo processamento no dispositivo IIoT (minimizando o $t_{C P U}$ ).

\subsection{Método de Compressão de Dados}

Uma abordagem adequada para minimizar a quantidade de dados transmitidos, permitir a redução do MTU e, consequentemente, o consumo de energia dos dispositivos IIoT é a compressão de dados. Além disso, essa compressão de dados precisa ser de baixa complexidade para minimizar o tempo de processamento da IIoT, reduzindo também o consumo de energia.

Neste artigo, apresentamos um método de compressão de dados de baixa complexidade para reduzir a quantidade de dados a serem transmitidos, com o objetivo de minimizar o consumo de energia de dispositivos IIoT com base na tecnologia IEEE 802.11 / WiFi. O método proposto é baseado na Codificação de Huffman, que possui uma alta taxa de compressão e baixo processamento de energia (complexidade computacional $O(n \log n)$ ) [Malleswari et al. ]. Essas características tornam a Codificação de Huffman uma abordagem adequada para dispositivos IIoT.

O método proposto aplica uma tabela de códigos fixa, apresentada na Tabela 2, para compactar e descompactar dados, onde a compressão ocorre no dispositivo IIoT de origem antes de enviá-lo por uma rede Wifi.

Uma visão geral do método de compressão de dados proposto é ilustrada na Figura 1, onde também é apresentado um exemplo prático. Primeiro, as informações dos dispositivos IIoT são coletadas para serem convertidas em dados binários (usando a estrutura definida na tabela 2), resultando em uma faixa binária.

Posteriormente, a faixa binária é dividida em uma matriz de 6 bits, onde cada número gera uma posição na matriz. São adicionados 01 no início de cada posição da matriz. Em seguida, cada posição da matriz é convertida em um caractere e os grupos de caracteres em uma sequência (dados compactados). Finalmente, a sequência é enviada pela interface IEEE 802.11 / WiFi do dispositivo IIoT. 
Tabela 2. Codificação de Huffman para o cenário de teste

\begin{tabular}{|c|c|}
\hline Número & Codificação de Huffman \\
\hline \hline 0 & 111 \\
\hline 1 & 101 \\
\hline 2 & 000 \\
\hline 3 & 0101 \\
\hline 4 & 0100 \\
\hline 5 & 0111 \\
\hline 6 & 0011 \\
\hline 7 & 1000 \\
\hline 8 & 1001 \\
\hline 9 & 0110 \\
\hline- & 0010 \\
\hline$\cdot$ & 110 \\
\hline
\end{tabular}

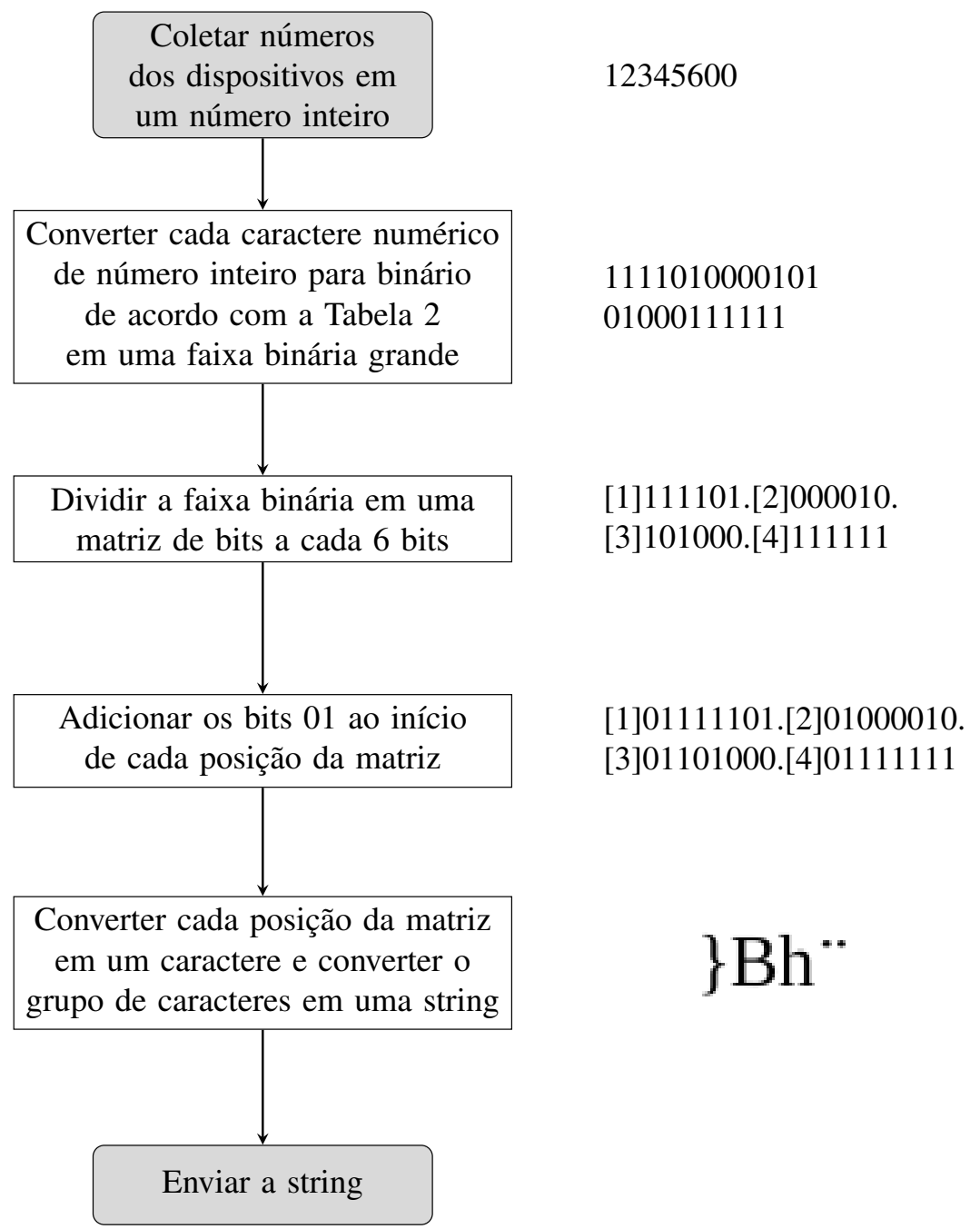

Figura 1. Método de Compressão de Dados. 
É válido ressaltar que as técnicas mais modernas de compressão tornam-se inviáveis para cenários de IIoT e serem implementadas no HW usado, onde podemos destacar Partial Matching [Bille et al. 2018], Golomb [Leon-Salas 2015] e Shannon-Fano [Mantoro et al. 2017]. Estas técnicas são baseadas em análise probabilística e predição, as quais necessitam de uma maior capacidade computacional (memória e CPU) para calcular a ocorrência dos caracteres e gerar um resultado ótimo, elevando muito a complexidade e tempo de processamento (consequentemente consumo de energia).

\section{Experimentos Realizados}

Esta seção apresenta os experimentos realizados para avaliar o método de compressão de dados proposto para IIoT. Para realizar os experimentos, foi implantado um cenário com dispositivos reais para avaliar os benefícios do método de compressão de dados proposto, bem como o impacto do tamanho do pacote, na vida útil da bateria dos dispositivos IIoT. A implementação do algoritmo de compressão proposto foi feita diretamente dentro do ESP8266X/WEMOS, visto que o mesmo não possui bibliotecas para suporte a tal (por isso faz-se necessário uma abordagem de baixa complexidade). A seção 4.1 apresenta a configuração do experimento e a seção 4.2 discute os resultados.

\subsection{Configuração do Cenário de Testes}

No cenário de teste montado foi usado dois hardwares ESP8266X / WEMOS [Kanakaris et al. 2019]. Esses dispositivos IIoT têm baixo custo financeiro e são compatíveis com IEEE 802.11 / WiFi b / g / n (HT20). Ambos os dispositivos funcionam com uma frequência de $80 \mathrm{M} \mathrm{Hz}$ ou $160 \mathrm{M} \mathrm{Hz}$ e podem ser alimentados com $3.3 \mathrm{~V}$ $0,5 \mathrm{~A}$. Durante os experimentos, usamos duas baterias 18650 com uma tensão nominal de $8,2 v-7,6$ e um módulo regulador de tensão de $3,3 \mathrm{~V}$ para reduzir a tensão para $3,3 \mathrm{~V}$ [Kanakaris et al. 2019]. Assim, as baterias usadas têm capacidade de $2080 \mathrm{mAh}$ e taxa de descarga automática de $0,52 \mathrm{~A}$ (com pico de 2,6A). A configuração dos experimentos é ilustrada na Figura 2.

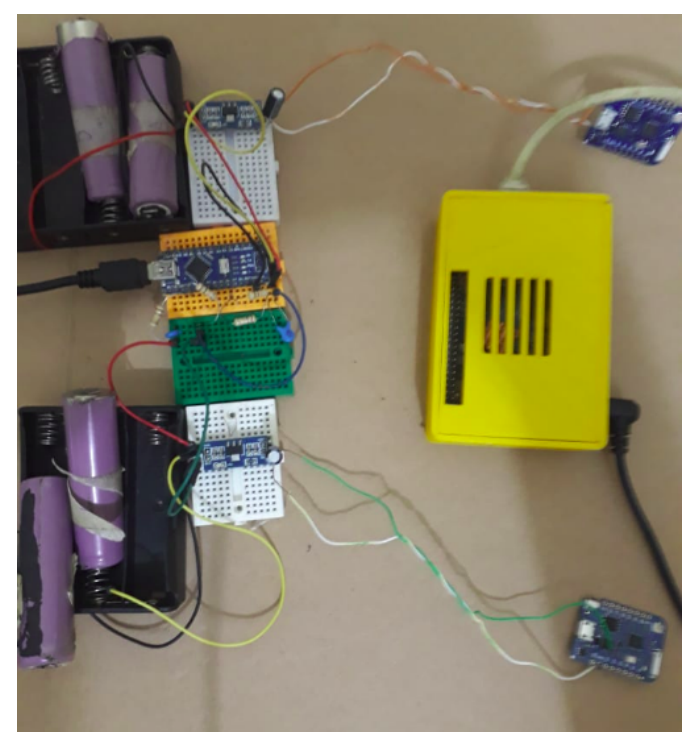

Figura 2. Cenário de Teste. 
O principal objetivo dos experimentos era medir o nível de tensão das baterias de acordo com o número de mensagens enviadas em um período específico, variando de $8 a$ 20 horas. Para coletar essas amostras de tensão da bateria, um arduino foi usado para obter 100 amostras em uma frequência de 10hz e registrar esses dados, onde o coletor de tensão entra em estado de suspensão por 60 segundos antes de reiniciar esse processo.

Durante os experimentos, os dois dispositivos ESP8266X / WEMOS usaram o padrão 802.11n com $20 \mathrm{MHz}$ de largura de banda, transmitindo dados simultaneamente. O protocolo MQTT foi usado como um aplicativo de mensagens para garantir a comunicação ponto a ponto, melhorando a análise e o registro de dados. O objetivo dessa configuração era avaliar o comportamento da variação de tensão e, consequentemente, a vida útil da bateria, de acordo com as abordagens de transmissão e compressão de dados. Os experimentos foram realizados 25 vezes, onde cada um coleta 80.000 amostras (cerca de 9 horas de duração).

Em relação à análise dos dados, foi avaliado o Nível de Tensão Normalizado (Equação 2) e a Inclinação da Regressão Linear dos dados coletados (Equação 3). A tensão foi normalizada para reduzir as distorções nos dados coletados, pois as duas baterias não possuem o mesmo nível de tensão. Na Equação 2, $\mu$ é a média da distribuição, $\sigma$ é a variação e $x$ é a variável independente. A Inclinação da Regressão Linear representa a tendência (ascendente ou descendente) em um vetor determinado por $y=a * x+c$. Em nossa análise, quando $b$ é negativo (tende ao eixo $x$ ), representa um maior consumo de energia.

$$
\begin{gathered}
f(x, \mu, \sigma)=\frac{1}{\sqrt{2 \pi \sigma}} e^{-\frac{(x-\mu)^{2}}{2 \sigma^{2}}} \\
b=\frac{\sum(x-\bar{x})(y-\bar{y})}{\sum(x-\bar{x})^{2}}
\end{gathered}
$$

\subsection{Resultados}

Esta seção apresenta os resultados dos experimentos, comparando o desempenho do método de compressão de dados proposto e a abordagem tradicional para IIoT. Portanto, avaliamos o impacto do método proposto na vida útil da bateria e transmissão de dados.

A taxa de compressão, em média, foi significativamente alta (80\%), permitindo a redução do MTU de 1000 (usado na abordagem tradicional) para 256 (usado na proposta). É válido ressaltar que a CPU dos dispositivos é extremamente limitada $(80 \mathrm{M} \mathrm{Hz}$ ou $160 \mathrm{MHz}$ ), sendo necessário o uso de uma técnica de compressão leve como a proposta deste trabalho, visto que a compressão será executada no dispositivos IIoT.

Dividimos em dois experimentos a análise de consumo de energia: a seção 4.2.1 descreverá os experimentos para avaliar os benefícios da minimização da transmissão de dados para economia de energia na tecnologia IEEE 802.11 / WiFi; e a seção 4.2.2 apresentará a comparação do consumo de energia entre a abordagem tradicional e o método proposto variando o MTU, respectivamente. 


\subsubsection{Avaliação da Transmissão de Dados}

Neste experimento um dispositivo IIoT enviou 500 bytes de mensagens e o outro dispositivo transmitiu 15 bytes de mensagens compactadas. Ambos os dispositivos usaram um MTU de 1000 bytes. O principal objetivo deste experimento foi medir o impacto do tamanho dos dados transmitidos no consumo de energia dos dispositivos IIoT, onde as figuras 3 e 4 apresentam os resultados.

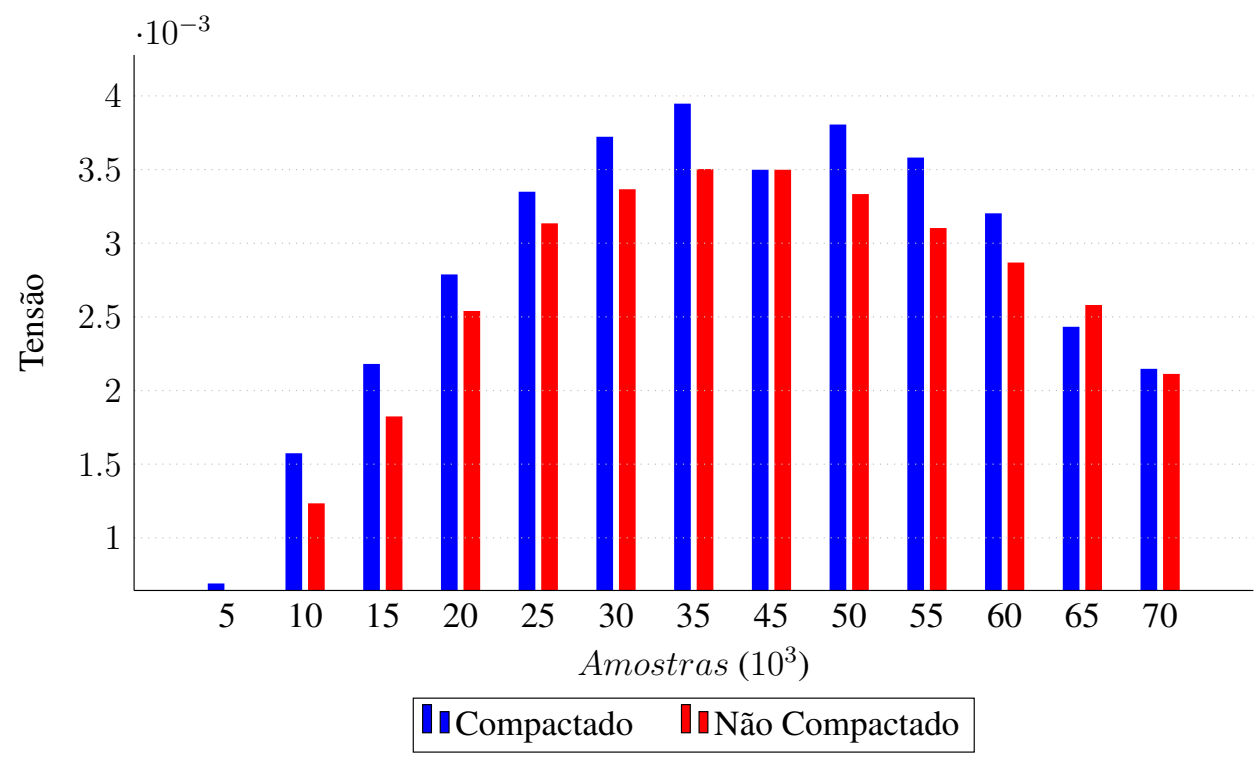

Figura 3. Tensão da Bateria Normalizada: 500 bytes (não compactado) e 15 bytes (compactado).

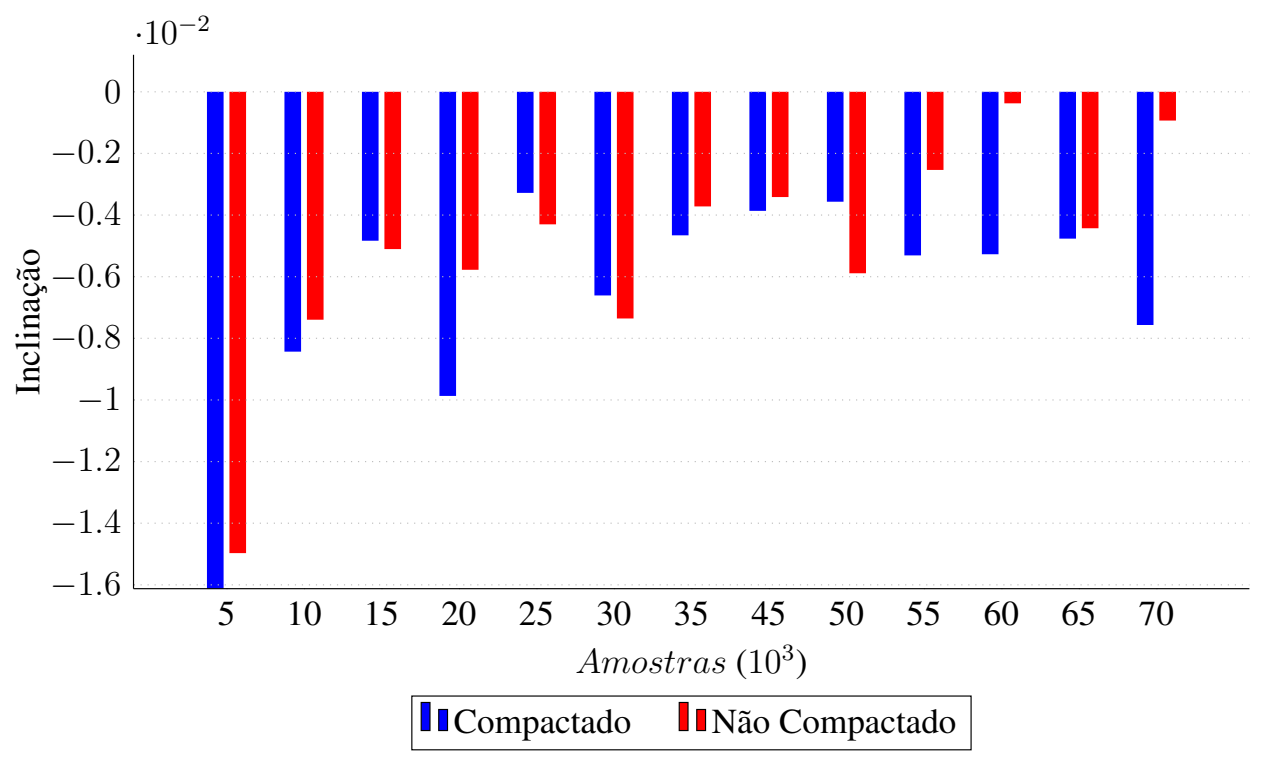

Figura 4. Inclinação da regressão do consumo de energia: 500 bytes (não compactado) e 15 bytes (compactado).

Com base nas informações da Figura 3, é possível observar que o envio de 500 bytes (descompactado) gastou mais energia do que 15 bytes compactados (variando de 7 
$\%$ a $42 \%$ durante o experimento), ou seja, o consumo de energia para enviar 500 bytes é maior do que para compactar os dados (processamento de dados da CPU) e enviar 15 bytes.

Da mesma forma, usando uma análise de inclinação da regressão linear apresentada na Figura 4, notamos que os dados compactados tiveram uma redução no consumo de energia de $8 \%$, devido à diferença entre o tamanho original e a mensagem compactada. Assim, observamos que o envio de mensagens maiores na tecnologia IEEE 802.11 / WiFi consome mais energia e reduz a vida útil das baterias.

\subsubsection{Comparação da Proposta}

Neste experimento, analisamos o impacto da redução do MTU no consumo de energia, avaliando os seguintes casos: (I) MTU de 1000 bytes sem compressão e (II) MTU de 256 bytes utilizando o método de compressão proposto. Enviamos mensagens de 300 bytes e mensagens de 137 bytes após a compressão. Portanto, esses experimentos avaliaram a capacidade do método de compressão de dados proposto para permitir a adequação do MTU, bem como seu impacto no consumo de energia. Os resultados são ilustrados nas Figuras 5 e 6.

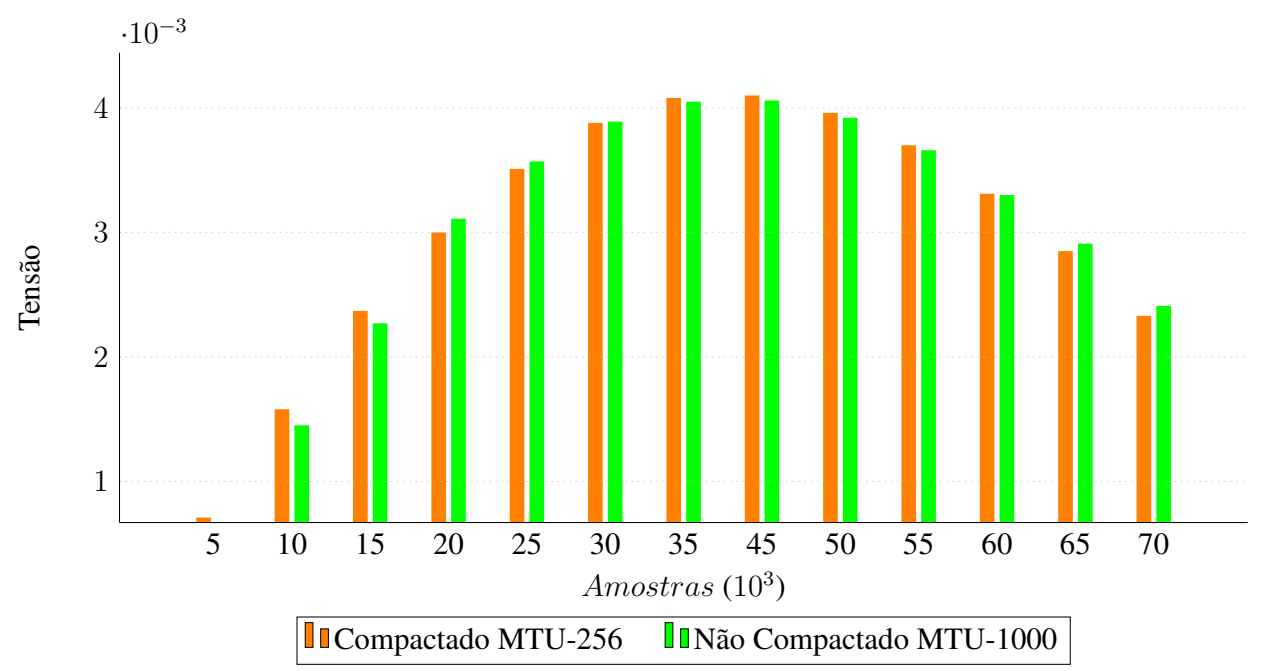

Figura 5. Tensão da Bateria: MTU-256 compactado e MTU-1000 Não Compactado.

A partir dos dados apresentados, é possível observar uma pequena alteração no consumo de energia dos dispositivos a partir da variação do MTU, tanto na análise da tensão bem como de inclinação da regressão linear. Portanto, percebe-se que mesmo adicionando uma carga extra de processamento para realizar a compactação, a solução proposta gera um consumo de energia similar ao a abordagem tradicional, mas trazendo consigo o benefício de redução do MTU, o qual melhorar a capacidade de acesso ao meio dos dispositivos IIoT e a escalabilidade da rede no contexto de Industria 4.0.

\subsection{Discussão}

A partir dos dados apresentados, é possível observar que o método proposto resultou em um ganho de vida útil da bateria (em torno de $8 \%$ ), tanto na análise da tensão bem como de inclinação da regressão linear. 


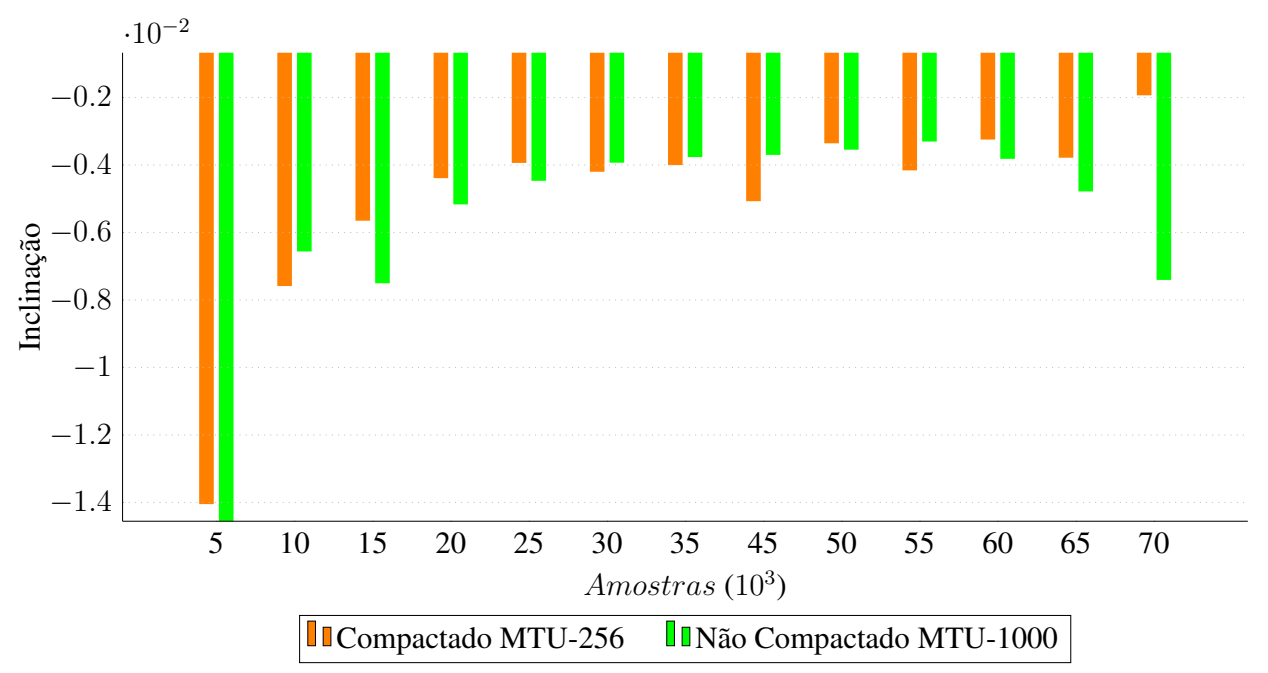

Figura 6. Inclinação da regressão do consumo de energia:: MTU-256 compactado e MTU-1000 não compactado.

É válido ressaltar que o tamanho do MTU interfere diretamente no acesso ao meio dos dispositivos, visto que o tamanho do pacote transmitido é diretamente relacionado ao tempo de ocupação do meio [Datasheet 2015, Siu 2018]. Por exemplo, com uma taxa de transmissão de 11Mbits leva-se 254 microsegundos para transferência de 350 Bytes, enquanto que gasta-se 145 microsegundos para transferência de 200 Bytes. Desta forma, a escalabilidade da rede pode aumentar em até aproximadamente $42 \%$ com uma redução deste MTU. O impacto da variação do MTU ficaria mais nítido em um experimento de maior escala.

Sendo assim, uma abordagem que minimize o gasto de energia com transmissão e habilite a redução deste MTU aproxima a aplicação de IEEE 802.11 / WiFi em IIoT em relação as soluções existentes. Estas soluções, como por exemplo 6LoWPAN [Shelby and Bormann 2011, Olsson 2014], trazem as vantagens de um MTU reduzido e consumo de energia baixo, mas há as desvantagens de baixa largura de banda e menor tempo de acesso em ambientes heterogêneos (por exemplo, 802.11 e 802.15.4) [Siu 2018].

De acordo com os resultados, a abordagem de compressão de dados proposta evolui a capacidade dos dispositivos IIoT de atender aos requisitos da indústria 4, 0 por meio da redução dos dados transmitidos e do algoritmo de compressão de baixa complexidade (pequeno consumo de energia e baixo processamento).

\section{Conclusão}

O crescente uso de dispositivos sem fio em ambientes industriais promoveu a aplicação da IIoT para atender aos requisitos da indústria de 4.0. Uma abordagem promissora para implantar ambientes IIoT é o uso da tecnologia IEEE 802.11 / WiFi, devido aos recursos de escalabilidade, segurança e padronização. No entanto, o uso da tecnologia IEEE 802.11 / WiFi precisa ser adaptado para melhorar sua eficiência energética.

Portanto, este artigo apresentou um método de compressão de dados para reduzir a quantidade de dados a serem transmitidos, com o objetivo de minimizar o consumo de energia dos dispositivos IIoT. O método proposto permite que os dispositivos IIoT configurem um MTU menor e, consequentemente, a energia consumida pelas transmissões 
sem fio, através de um algoritmo de compressão de baixa complexidade (reduzindo o consumo de energia e processamento).

O método de compressão de dados proposto foi avaliado em ambiente de teste real, composto por vários ESP8266 usando o protocolo MQTT, analisando o impacto da proposta em dispositivos IIoT. Os resultados sugerem que a proposta reduz o consumo de energia em $8 \%$ na frente dos ambientes tradicionais da IIoT. Como trabalho futuro, pretendemos analisar o desempenho usando o modo de sono profundo e baterias de baixa descarga interna por longos períodos. Além disso, pretende-se executar mais experimentos com um maior número de nós, possibilitando uma melhor avaliação do impacto do MTU em redes de grande escala.

\section{Referências}

Akintade, O. O., Yesufu, T. K., Kehinde, L. O., et al. (2019). Development of power consumption models for esp8266-enabled low-cost iot monitoring nodes. Advances in Internet of Things, 9(01):1.

Bille, P., Christiansen, A. R., Cording, P. H., Gørtz, I. L., Skjoldjensen, F. R., Vildhøj, H. W., and Vind, S. (2018). Dynamic relative compression, dynamic partial sums, and substring concatenation. Algorithmica, 80(11):3207-3224.

Biswas, S., Das, R., and Chatterjee, P. (2018). Energy-efficient connected target coverage in multi-hop wireless sensor networks. In Industry interactive innovations in science, engineering and technology, pages 411-421. Springer.

Björnson, E. and Larsson, E. G. (2018). How energy-efficient can a wireless communication system become? In 2018 52nd Asilomar Conference on Signals, Systems, and Computers, pages 1252-1256. IEEE.

Datasheet, E. (2015). Esp8266ex datasheet. Espressif Systems Datasheet, pages 1-31.

Davoli, L., Belli, L., Cilfone, A., and Ferrari, G. (2017). From micro to macro iot: Challenges and solutions in the integration of ieee 802.15. 4/802.11 and sub-ghz technologies. IEEE Internet of Things Journal, 5(2):784-793.

Ertaul, L. and Woodall, A. (2017). Iot security: Performance evaluation of grain, mickey, and trivium-lightweight stream ciphers. In Proceedings of the International Conference on Security and Management (SAM), pages 32-38. The Steering Committee of The World Congress in Computer Science, Computer ....

Kanakaris, V., Papakostas, G., and Bandekas, D. (2019). Power consumption analysis on an iot network based on wemos: a case study. Telkomnika, 17(5):2505-2511.

Leon-Salas, W. D. (2015). Encoding compressive sensing measurements with golombrice codes. In 2015 IEEE International Symposium on Circuits and Systems (ISCAS), pages 2177-2180.

Li, K., Yuen, C., Kusy, B., Jurdak, R., Ignjatovic, A., Kanhere, S. S., and Jha, S. (2018a). Fair scheduling for data collection in mobile sensor networks with energy harvesting. IEEE Transactions on Mobile Computing, 18(6):1274-1287.

Li, Z., Liu, Y., Liu, A., Wang, S., and Liu, H. (2018b). Minimizing convergecast time and energy consumption in green internet of things. IEEE Transactions on Emerging Topics in Computing. 
Long, N. B., Tran-Dang, H., and Kim, D.-S. (2018). Energy-aware real-time routing for large-scale industrial internet of things. IEEE Internet of Things Journal, 5(3):21902199.

Malleswari, M., Krishna, B. A., Madhuri, N., and Chowdary, M. K. Implementation of modified huffman coding in wireless sensor networks. International Journal of Computer Applications, 975:8887.

Mantoro, T., Ayu, M. A., and Anggraini, Y. (2017). The performance of text file compression using shannon-fano and huffman on small mobile devices. In 2017 International Conference on Computing, Engineering, and Design (ICCED), pages 1-5.

Mesquita, J., Guimarães, D., Pereira, C., Santos, F., and Almeida, L. (2018). Assessing the esp8266 wifi module for the internet of things. In 2018 IEEE 23rd International Conference on Emerging Technologies and Factory Automation (ETFA), volume 1, pages 784-791.

Mumtaz, S., Alsohaily, A., Pang, Z., Rayes, A., Tsang, K. F., and Rodriguez, J. (2017). Massive internet of things for industrial applications: Addressing wireless iiot connectivity challenges and ecosystem fragmentation. IEEE Industrial Electronics Magazine, 11(1):28-33.

Olsson, J. (2014). 6lowpan demystified. Texas Instruments, 13.

Sharma, P. K., Jeong, Y., and Park, J. H. (2018). Eh-hl: Effective communication model by integrated eh-wsn and hybrid lifi/wifi for iot. IEEE Internet of Things Journal, 5(3):1719-1726.

Shelby, Z. and Bormann, C. (2011). 6LoWPAN: The wireless embedded Internet, volume 43. John Wiley \& Sons.

Sisinni, E., Saifullah, A., Han, S., Jennehag, U., and Gidlund, M. (2018). Industrial internet of things: Challenges, opportunities, and directions. IEEE Transactions on Industrial Informatics, 14(11):4724-4734.

Siu, C. (2018). IoT and Low-Power Wireless: Circuits, Architectures, and Techniques. CRC Press.

Talebi, M., Papatsimpa, C., and Linnartz, J.-P. M. (2018). Dynamic performance analysis of ieee 802.15. 4 networks under intermittent wi-fi interference. In 2018 IEEE 29th Annual International Symposium on Personal, Indoor and Mobile Radio Communications (PIMRC), pages 1-7. IEEE.

Tömösközi, M., Seeling, P., Ekler, P., and Fitzek, F. H. (2017). Robust header compression version 2 power consumption on android devices via tunnelling. In 2017 IEEE International Conference on Communications Workshops (ICC Workshops), pages 418-423. IEEE.

Varghese, A. and Tandur, D. (2014). Wireless requirements and challenges in industry 4.0. In 2014 International Conference on Contemporary Computing and Informatics (IC3I), pages 634-638. IEEE.

Wollschlaeger, M., Sauter, T., and Jasperneite, J. (2017). The future of industrial communication: Automation networks in the era of the internet of things and industry 4.0. IEEE industrial electronics magazine, 11(1):17-27. 